\title{
Development and characterization of a multi-platform Data Acquisition System for Power Quality metrological certification
}

\author{
M. Caciotta, S. Giarnetti, G. Lattanzi Cinquegrani, F. Leccese and D.Trinca \\ Department of Electronic Engineering \\ Roma Tre University \\ Via della Vasca Navale n.84, 00146, Rome (Italy) \\ Phone/Fax number:+39 06 57337342, e-mail: caciotta@uniroma3.it, s.giarnetti@uniroma3.it, \\ gia.lattanzicinqu@uniroma3.it, leccese@uniroma3.it, daniele.trinca@tin.it
}

\begin{abstract}
A Data Acquisition USB Device has been designed, developed, realized and characterized to be used in Power Quality monitoring activities. The system simultaneously manage 8 data acquisition channels to allow acquisition on tri-phases plus neutral lines working at 499 samples per $50 \mathrm{~Hz}$ period. It can get external certified time and voltage references to warranty certified measurements. The system is directly powered by USB interface and use a multiplatform FTDI Virtual ComPort drivers to yield a simpleto-use and scalable device able to send and receive data over High Speed RS232 virtual interfaces, without requiring any advanced programming skill. The virtual serial communication makes data acquisition software portable over many platforms, regardless development environment and programming language. The proposed device has been tested with custom software written in C\# and VB6, than in LabView and MatLab environment, moreover it has been characterized applying the most common ADC performing test to verify its behaviour.
\end{abstract}

\section{Key words}

Power Quality, Acquisition Card, MAX1320, Multiplatform System.

\section{Introduction}

The constant grow up of non linear loads connected to the electric network is the principal cause of the necessity to measure the electrical energy quality. These loads produce a series of variations on ideal current and voltage waveform that have to be studied and monitored to preserve the network, the generators and the loads by possible breakings. Power Quality is the branch of the science that analyzes these alterations[1] covering a multitude of types of power system disturbances and studying the interactions that can appear in a generic electric network between generators and loads. The CEI EN 50160 and subsequent are the norm that defines all the characteristics of the electricity supplied by public distribution systems [2].

In order to study the Power Quality it is necessary to measure the normed parameters using proper instrumentation, as suggested in CEI IEC 61000-4-7 [3]. In the market there are many instruments that measure Power Quality[4] but they have several order of problems as, for e.g.:

1) to obtain high accuracy measurements the instruments' costs are high[5];

2) often, the sensors are not adequate to PQ needs, as, e.g. the use of ferromagnetic core sensors with pass band too small that don't allow to correctly measure the harmonic and interharmonic content;

3) normally, the market instruments are not designed to obtain automatic measurements for long time cause the little memory deep;

4) multiple channels acquisition cards use a multiplexed management for the input channels, this produce an incorrect registration of the electric phases under analysis $[6,7,8]$;

5) the acquisition trigger is not connected to a metrological time reference making the metrological approach toward the measurements fruitless, or, where it is verified, the multiplexing is managed by a local quartz that has a poor time accuracy $[6,7,8]$;

6) devices designed for automatic stand alone measurements have not yet a high reliability[6,7,9].

These lasts three problems have been directly found on instrument realized by ourselves, partially solved with a complex uncertainty determination (problem 4) [6] or with suitable recovery remote control system (problem 6) $[8,9]$.

To face part of these problems it has been developed an "ad hoc" Data Acquisition System (DAS).

We improved performances of the previous measurement instrumentation, widely described in $[6,7,8]$, developing a custom acquisition board based on high speed USB 2.0 interface, capable to acquire simultaneously 8 channels working at $24.976 \mathrm{kHz}, 14$ bit word depth, that guarantees 499 samples per AC cycle, over the lower boundaries of 256 samples, recommended by emerging standards like IEC 61850[10].

The DAS system is equipped with the versatile FT2232HQ USB interface whose drivers are available for Windows $\bigodot$, Linux and MacOSX operating systems and represents a wide range of opportunities for DAS to interface with existing platforms, both hardware and software.

The board has its own time and voltage reference on; when available, it's possible to set up external references for metrological purposes. In fact, the board has designed to be connected directly to a certified $2,048 \mathrm{kHz}$ timing signal provided by Telecom Italia and synchronized with atomic clock of INRIM institute, holder of national legal time counting.

System control is quite easy via Virtual Com Port (VCP) driver with a very simple command set over a bidirectional 9,600 bps null modem channel. A second VCP high speed one-way channel is dedicated to transmit captured data over USB connection. The software driver 
manages both control and data channel like a RS232 COM port. This method simplifies the development of cross-platform capture and processing software, without any advanced programming skill.

\section{Proposed Instrument}

The proposed solution aims to be a universal cross platform interface for all those hardware equipped with USB 2.0 interface and able to mount a well known operating system like: Windows $\bigodot$ family products, Linux distributions and Mac OSX. The most part of today available hardware platforms have a USB interface on board and can support an operating system like those listed above, in standard or embedded version. Using FT2232HQ device rather than PCI interface let us to get important advantages in terms of software and hardware compatibility, including every notebook, PDA devices and most ARM processor embedded machines.

On the other hand many PCI based commercial data acquisition devices often make use of own SDK (software development kit) package for board control applications, usually released in inaccessible precompiled program libraries. In many cases user must have an advanced programming skill level in ANSI $\mathrm{C}$ or $\mathrm{C}++$ languages to write his own custom data acquisition software. Another approach for board control is represented by painless but expensive software packages like LabView (C) usually used for prototyping and testing purposes due to licensing costs.

Our DAS is designed to be as simple as possible to be used by every common user who wants to focus his attention to research and data management instead low level programming. In fact FTDI drivers allow to control the board using a standard serial communication across two dedicated Virtual COM ports (VCP), in RS232 emulation way: the first one is a low speed 9,600 bps communication to send control data and receive current status; the second is a high speed "one way" receiver channel for acquired data.

DAS system results versatile to use on all hardware because it's an external self powered device over USB bus, with a power consumption of $300 \mathrm{~mA}$; furthermore it's easy to write custom data acquisition software accessing serial COM ports. The most of high level languages like (Visual Basic, C\#, Java and so on) support natively serial port communications, and make it possible to realize open source data managing software without any licensing constraints.

We realized for testing a custom C\# 2005 software running on .NET framework 2.0 and Windows XP, then the device has been tested with a VB6 application under Windows 2000 too, and finally in MATLABC and LabVIEW@ environments.

\section{Usb Data Acquisition Device Architecture}

The USB data acquisition device has been structured with the following blocks: (a) USB interface, (b) sample references, (c) control logic, (d) data acquisition core, (e) input channels. The DAS' block scheme is reported in figure 1 .

a) USB interface. Handles data $\mathrm{I} / \mathrm{O}$ communication upon a pair of channel between data acquisition board and the host controller: a PC in our case. Actually the interface is implemented through an FTDI FT2232HQ Mini Module EV-KIT[11]. A mini-USB plug connector is mounted on the evaluation kit that fits 2 internally independent UART/FIFO ports, configurable in external EEPROM over the USB interface. The device has been configured to operate the FT2232HQ in dual high speed USB to multipurpose UART/FIFO. The first channel, labeled as "A", has been configured as 8 bit wide parallel FIFO asynchronous interface (FT245B mode), is able to receive acquired data up to $8 \mathrm{Mb} / \mathrm{s}$; the second, the "B" channel, has been configured as a standard bidirectional RS232 COM port, 9,600 bps, 8 data bits, no parity bit, and one stop bit encoding. "B" channel is dedicated to work as USB bridge for a PIC16F628A microcontroller that manages data acquisition control.

b) Sample references. The device is provided with ADC internal onboard calibrated and compensated $2.5 \mathrm{~V}$ voltage sample reference that fully meets the design specifications. High accuracy timing reference is externally provided to the board, incoming from Telecom Italia 2,048 $\mathrm{kHz}$ balanced signal and synchronized with atomic clock of INRIM institute, the Italian Metrological Institute. External references give metrological validity to the samples acquired from the device. The $2,048 \mathrm{kHz}$ signal is adapted with proper circuits to be suitable with electronics of device. Optional internal timing references is included in the project; it is realized with a $4,096 \mathrm{kHz}$ oscillator.

c) Control Logic. All control logic has been implemented into an Altera EPM240 CPLD (in Evaluation Kit)[12], using Quartus II 9.0 software and JTAG programmer[13]. This device performs the following activities: (a) divides $2,048 \mathrm{kHz}$ clock to obtain $26.976 \mathrm{kHz}$ sampling clock, and spread it to ADC when required; (b) manages ADC control signals for acquiring and data reading sequences; (c) splits 14 bits incoming parallel data from ADC into two words of 8 bits each one ( 8 bits plus 6 and 2 dummy bits); (d) writes split data on USB FIFO channel and sends 2 control bytes as "end of packet" terminator for redundancy purposes. EPM240 internal registers are directly controlled by a PIC 16F628A microcontroller[14]; it receives command from USB RS232 channel and sets up EPM240 register to perform following actions: counters reset, acquire a fixed number of samples (using 16 bit register), freerun acquisition, start and stop acquisition.

d) Data acquisition core. The data acquisition has com- 


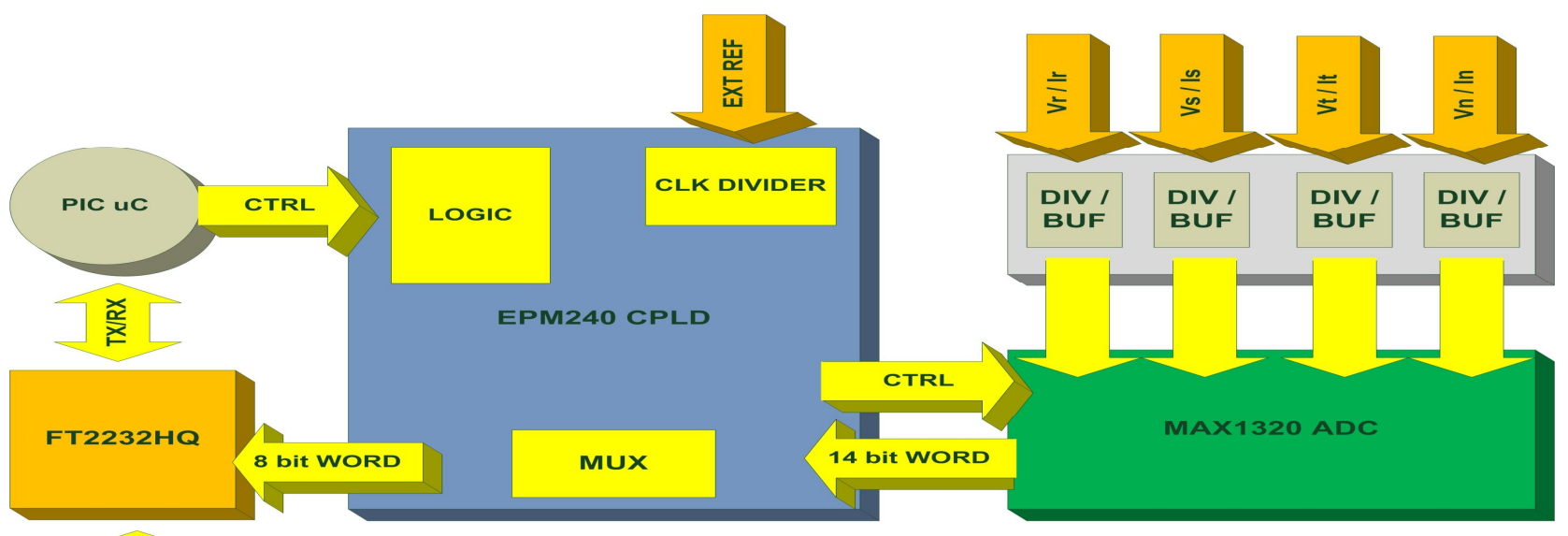

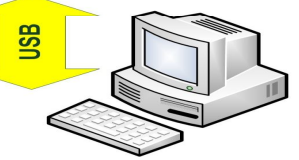

mitted to a Maxim MAX1320 ADC[15], working on it's original Evaluation Kit, provided with all the necessary electronic for optimal operating setup. This device is a very fast SAR ADC, capable to acquire simultaneously 8 channels at $250 \mathrm{kS} / \mathrm{s}$ per channel at 14 bits. It has an on chip "Track and Hold" circuit for each channel that guarantees $10 \mathrm{~ns}$ aperture delay and 50 ps Channel-To-Channel Track and hold matching. Analog input ranges are +/- $5 \mathrm{~V}, 77 \mathrm{~dB}$ SNR at 100 $\mathrm{kHz}$. It's very important to underlie that the 8 channels are acquired at the same time, in order to preserve all the information phases between voltages and currents. We configured the ADC to work always on 8 channels, in "reading after conversion" mode.

e) Input channels. MAX1320 Evaluation Kit is already equipped with 8 high performances input buffers on board (MAX4351); however we preferred to use different input buffers (LM6484[16]) to reduce total power consumption over USB port. Voltage inputs are scaled down with high precision decade dividers to be suitable with ADC inputs, with a tolerance of $0.01 \%$ (figure 2); then the scaled signal is buffered to $\mathrm{ADC}$, and it can so bear peaks up to $500 \mathrm{~V}$. Current signals instead arrive from an integrator realized by Rocoil Ltd, that allow us to integrate the signal incoming from the Rogowsky coils. Voltage and current channels are disposed alternately and spaced on the board to reduce as more as possible cross talking noise and capacitive couplings.

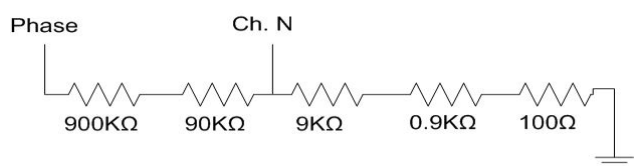

Fig. 2 Decade Dividers.

\section{Realized Circuits}

Figure 3 shows the singular blocks of the realized USB data acquisition device connected by wires. Figure 4 shows the PCB card (top (a), bottom (b)) that allows the integration on a single card of the singular parts composing the DAS.

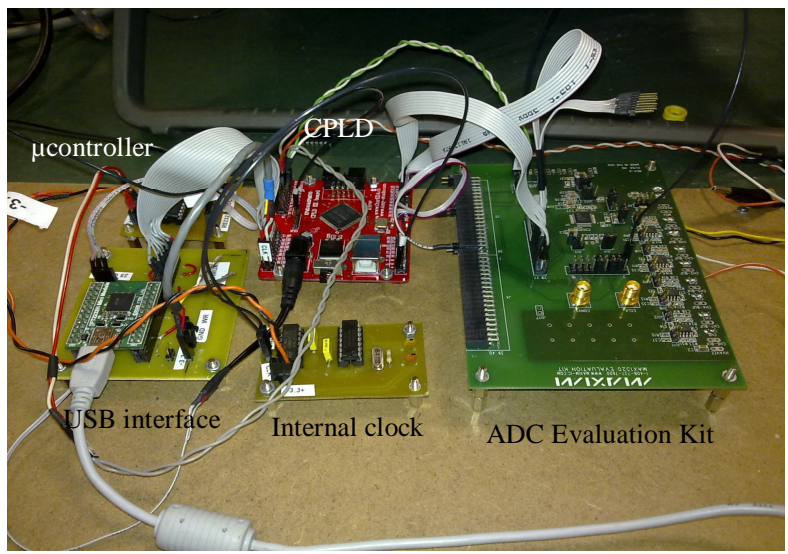

Fig. 3 The new acquisition card for PQ measurements.

This integration allows to eliminate the use of evaluation kits so optimizing costs and physical dimensions and makes more easy the assembling.

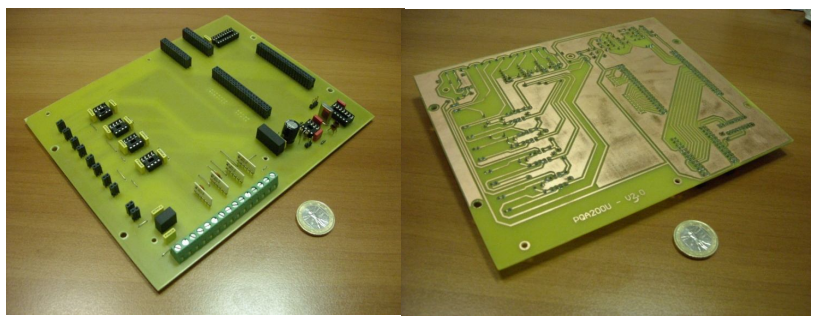

(a)

(b)

Fig. 4 The PCB card used for the integration.

Figure 5 shows the whole acquisition card inserted inside its box ready to be directly used on field.
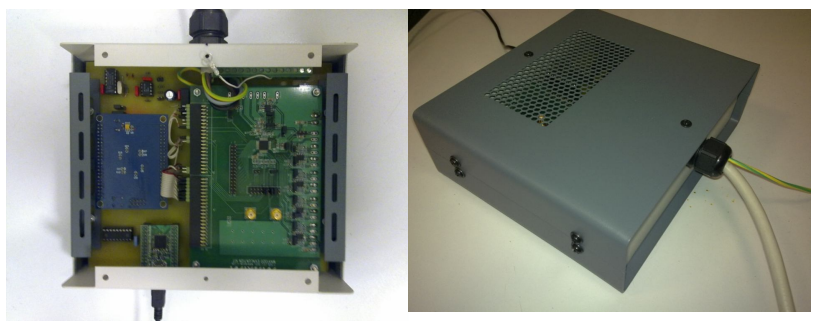

Fig. 5 The acquisition card in its box. 


\section{DAS Calibration}

To certify the measurements, it has been necessary to calibrate the A/D converter. The calibration procedure yields the use of a calibrated current and voltage waveform generator directly connected to the ADC's evaluation board. The calibrator used to generate these signals is the Fluke 5500A. The signals is sampled by the ADC and transferred to a PC by the USB interface. The calibrator is driven by the same PC through a virtual RS232. The management of the I/O data flux is obtained by a program written in Labview@ environment able, at the same time, to send commands and to store the samples.

Figure 6 shows the calibration bench where is possible to identify the calibrator, the acquisition card and the PC.

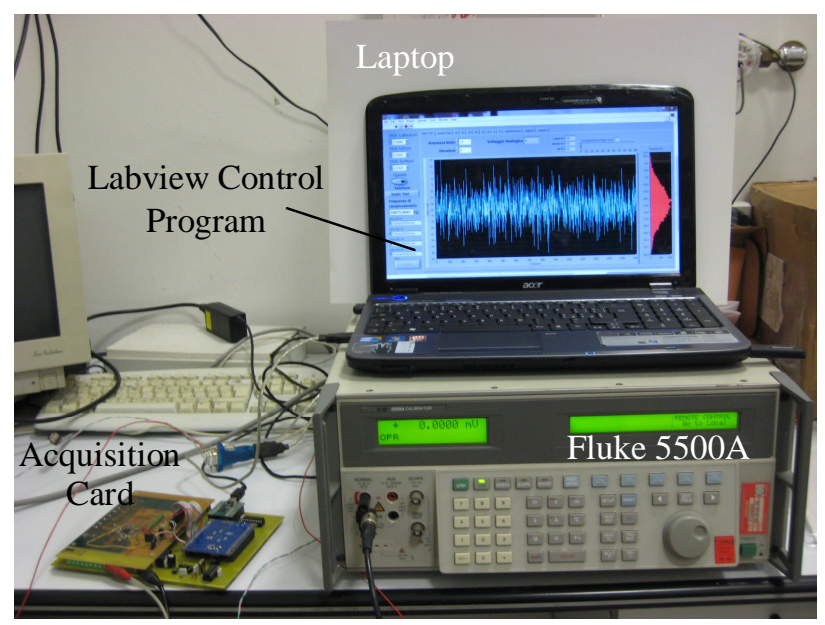

Fig. 6 The acquisition card calibration bench.

In order to calibrate the ADC we used two different tests: the first, called histogram test [17], is used to determine static parameters through a statistical analysis, the second, called sinewave curve fitting method [18], allows to determine dynamic parameters.

The static parameters analyzed are: offset, error gain and missing code.

The dynamic parameters analyzed are: SINAD, THD+Noise, ENOB, frequency, phase and amplitude of the sinusoid input signal.

\section{A. Static test}

To determine the static parameters has been used the ADC histogram test that involves collecting a large number of digitized samples over a specified period of time, for a well-defined input signal with a known probability density function. The ADC transfer function is then determined by a statistical analysis of the samples. During the test, 1500 samples have been acquired for each code bin of the ADC dynamic $(-5 \mathrm{~V} \div+5 \mathrm{~V})$; then the histogram has been determined collecting the number of occurrences of each code.

Applying the Pearson's chi-square test it has been possible to verify that the distribution of each code is normal with a confidence level of 0.95 .
The Histogram is an useful method for measuring the ADC input-referred noise, that is considered to be simply the standard deviation $(\sigma)$ of the distribution and is expressed in LSBs rms. Offset and gain were calculated as the mean of the distribution of the mid-scale code and the fitted characteristic of the mean of each distribution.

Figure 7 shows an example of the distribution of the 8,192-th code bin, that is the mid-scale code, and clearly points out that the ADC offset is about 17 bin equal to $10.3 \mathrm{mV}$.

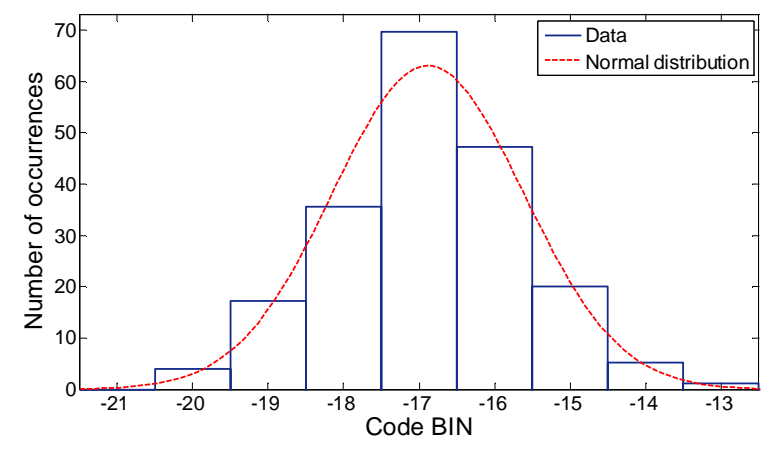

Fig. 7 Histogram and fitting of 8,192-th code bin distribution.

The presence of "wide codes," "narrow codes" and "missing codes" are easily spotted on the cumulative histogram by tallying the number of occurrences of each code in the full dynamic range of all levels.

\section{A. Dynamic test}

This method calculates a sinewave $\hat{x}[\mathrm{n}]$ that best fits the sample record using a square error minimization criterion. This calculation is performed by solving some nonlinear equations through an iterative algorithm. The fitting error (residue) is then calculated after the difference between the data record and the best fitting sine wave $\hat{\eta}[n]=y[n]-\hat{x}[n]$ and used to characterize the performance of the DAS board. The actual squared error is given by:

$$
\sigma_{\eta}^{2}=\sum_{n=1}^{M}\left[y[n]-A \cos \left(\omega \cdot t_{n}+\phi\right)-C\right]^{2}
$$

where $y[n]$ represents the data sample record, and $A, \omega, \varphi$ and $\mathrm{C}$ are the fitted parameters of, respectively, amplitude, frequency, phase and offset. To find the best fitting sinewave the values of $\mathrm{A}, \omega, \varphi$ and $\mathrm{C}$ have to be found.

We can retrieve additional informations from the residuals of a specific sinewave test. In particular, a user can determine the sources of error that are responsible for an ENOB that is less than expected. The residuals can be studied in the time domain and this representation is called the modulo time plot and is in detail described by Irons and Hummel [19]. To construct the modulo time plot, from the time coordinate of each data point in the record, is calculated the phase value between 0 and $2 \pi$, relative to the input sinewave, determined after the fitting procedure. This phase angle, divided by $2 \pi$, is shown on 
the horizontal axis of the plot. The residual value is shown on the vertical axis. The units are LSB. A scaled replica of the fitted input signal is displayed as the continuous curve. An example is shown in Figure 8.

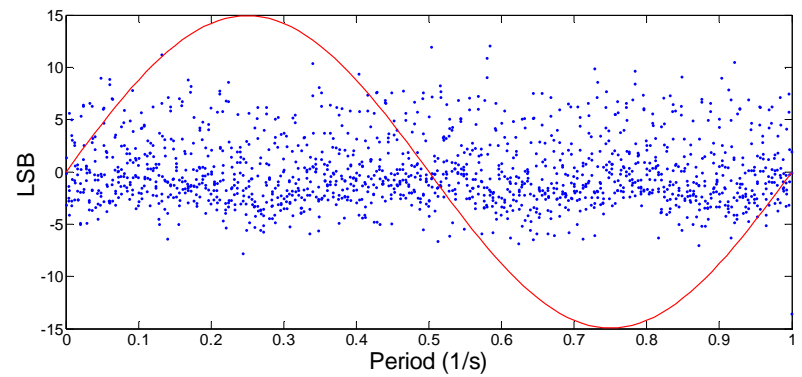

Fig. 8 Modulo time $(2 \pi)$ plot of the residuals versus phase angle.

On this plot, a user can clearly see random noise of about \pm 5 LSBs. The plot clearly displays the absence of phase relationship of the harmonic to the input signal. Unlikely the graph would show the phase relationship of the harmonic to the input signal, by presenting its negative peaks at the peaks of the input signal and positive ones at the zero crossing.

From dynamic test some other parameters that well describe the performance of the DAS under test have been also determined. In particular we determined:

1) SINAD: Signal-to-Noise-and-Distortion is the ratio of the rms signal amplitude to the mean value of the rootsum-square (rss) of all other spectral components, including harmonics, but excluding dc. SINAD is a good indication of the overall dynamic performance of an ADC because it includes all components which make up noise and distortion. Figure 9 shows SINAD parameter results for each DAS channel.

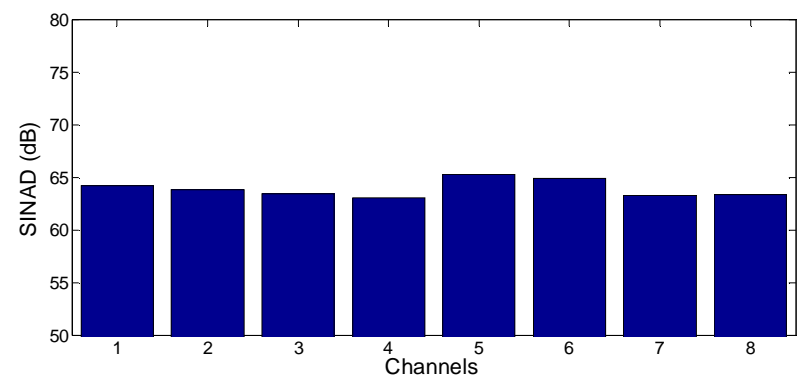

Fig. 9 SINAD.

2) THD+Noise: This parameter is the ratio, expressed in $\mathrm{dB}$ or in percentage, of the rms signal amplitude to the mean value of the root-sum-square (rss) of all other spectral components plus all spectral noise componets.
The bandwidth over which the noise is measured must be specified.

3) ENOB (Effective Number Of Bits): for an input sinewave of specified frequency and amplitude, ENOB is the number of bits of an ideal waveform recorder for which the rms quantization error is equal to the rms noise and distortion of the waveform recorder under test [17].

Table 1 shows the value of each parameter for each channel obtained from 1,500 samples of the input calibrated sinewave.

\section{Conclusion}

A metrologically certified Power Quality measurements data acquisition device has been realized and calibrated. It is able to accept external certified timing and voltage references. The device can simultaneously acquire data from 8 channels, at 499 samples per $50 \mathrm{~Hz}$ period with 14 bit amplitude resolution, well over the minimum ranges imposed by actual normative.

Acquired data can be sent to a compatible USB 2.0 hardware like a desktop PC, a PDA or embedded system supporting Windows, Linux or Mac OSX operating systems so realizing a multiplatform device.

It's very simple to write custom data acquisition software, because the system uses VirtualComPort drivers; user can access to the USB device taking advantages from high level programming languages, sending and receiving data on a standard RS232 COM port without the need of any kind of SDK or DLL library. The DAS is an USB self powered device, so it does not need any external power supply and can be connected directly to a standard $500 \mathrm{~mA}$ USB port. Joined to a notebook, it could be an ideal DAS able to operate in medium term black-out situations, without any UPS.

The USB device has been realized using existing hardware on evaluation kits, in order to reduce costs and PCB prototyping difficulties.

Acquisition software has been written in C\# 2005 to test USB device; it performs data acquisition using VCP drivers, stores data on hard disk and displays current graphic data on screen.

Has been designed a specific LabVIEW@ control to get data directly from the device. It's no needed any high performances PC to get USB device working; it was tested with success with a $1.2 \mathrm{GHz}$ Nehemiah MiniITX platform with Windows 2000@ and on a Core2Duo notebook running Windows 7 (C)

The realized USB Data Acquisition Device reduced the total costs of entire system; it has been proved to be

Table I. - Calculated dynamic ADC parameters

\begin{tabular}{|l|l|c|c|c|c|c|c|c|}
\hline & $\mathrm{CH} 1$ & $\mathrm{CH} 2$ & $\mathrm{CH}$ & $\mathrm{CH} 4$ & $\mathrm{CH} 5$ & $\mathrm{CH6}$ & $\mathrm{CH} 7$ & CH8 \\
\hline DC Offset (LSB) & 15.25 & 17.58 & 17.91 & 15.14 & 18.26 & 15.31 & 14.85 & 15.32 \\
\hline Amplitude (Vp) & 4.51 & 4.50 & 4.49 & 4.49 & 4.49 & 4.50 & 4.51 & 4.50 \\
\hline Frequency (Hz) & 49.99 & 50.00 & 50.00 & 50.01 & 49.99 & 50.00 & 50.01 & 50.01 \\
\hline Phase (deg) & 139.71 & 70.12 & 25.32 & 211.31 & 321.56 & 45.36 & 89.22 & 186.87 \\
\hline Effective Bits & 10.36 & 10.30 & 10.24 & 10.18 & 10.54 & 10.48 & 10.22 & 10.23 \\
\hline Noise + distortion (LSB) & 0.06 & 0.06 & 0.06 & 0.07 & 0.05 & 0.05 & 0.05 & 0.05 \\
\hline Sinad (dB) & 64.16 & 63.77 & 63.42 & 63.05 & 65.25 & 64.88 & 63.30 & 63.35 \\
\hline
\end{tabular}


competitive with actual data acquisition boards in terms of costs and performances.

Finally it was implemented a calibration process to characterize the system and prove the matching performances with test data.

The next project step will be the full integration of singular parts composing the actual DAS board, eliminating the use of evaluation kits, to further optimize costs, performances and physical dimensions.

\section{References}

[1] R.C. Dugan, M.F. McGranaghan, S. Santoso, H.W. Beaty, Electrical Power Systems Quality - second edition, McGraw - Hill, 2004.

[2] CEI EN 50160: Voltage characteristics of electricity supplied by public distribution systems.

[3] IEC 61000-4-7, (1991). Testing and measurement techniques - general guide on harmonics and interharmonics measurements for power supply systems and equipments connected thereto.

[4] Internet information about PQ Analyzers of several producers.

[5] F. Leccese: "Electrical Energy Measurements for Rome LV Customers by Distributed Web-Server Instruments" presented to $8^{\text {th }}$ EEEIC International Conference on Environment and Electrical Engineering (IEEE sponsored), May 10-13, 2009, Wroclaw, Poland, Proceedings, ISBN: 978-3-940471-10-9.

[6] M. Caciotta, F. Leccese, T. Trifirò: "From Power Quality To Perceived Power Quality" presented to The IASTED International Conference on Energy and Power Systems EPS 2006, 29-31 March 2006, Chiang Mai, Thailand, Proceedings 526-119, pp. 94-102, ISBN CD: 0-88986586-8.

[7] F. Leccese: "Rome: A First Example of Perceived Power Quality of Electrical Energy" presented to The Seventh IASTED International Conference on Power and Energy Systems EuroPES 2007 , August 29-31, 2007, Palma de Mallorca, Spain, Proceedings 582-084, pp. 169-176, ISBN Hardcopy: 978-0-88986-689-8 / CD: 978-0-88986690-4.

[8] F. Leccese: "Analysis of Power Quality Data on some Telecommunication Sites in Rome" presented to The Eight IASTED International Conference on Power and Energy Systems EuroPES 2008 , June 23-25, 2008, Corfù, Greece, Proceedings 608-086, pp. 62-67, ISBN CD: 978-0-88986-729-1.

[9] F. Leccese: "Study and Characterization of a New Protection System Against Surges and Over Voltages for Domestic Telecommunication Networks" presented to International Telecommunications Energy Conference INTELEC 2007, September 30 - October 4 2007, Rome, Italy, Proceedings pp. 363-368, ISBN: 978-1-4244-16288, IEEE Catalog Number: CFP07INTC, Library of Congress: 88-656128.

[10] IEC 61850. Communication Networks and Systems in substations.

[11] FT2232H Mini Module - USB Hi- Speed FT2232H Evaluation Module Datasheet.

[12] Altera EPM240 CPLD Datasheet.

[13] Altera Quartus II 9.0 software Application Notes.

[14] MICROCHIP PIC 16F628A microcontroller Datasheet.

[15] Maxim MAX1320 ADC Datasheet.

[16] LM6484 Datasheet.

[17] W. Kester, "Analog-Digital Conversion," Analog Devices press, 2004.
[18] D. Dallet and J. Machado da Silva, (eds.), "Dynamic Characterisation of Analogue-to-Digital Converters,", 105-156. (C) 2005 Springer. Printed in the Netherlands.

[19] F.H. Irons, D.M. Hummels, "The Modulo Time-Plot- A Useful Data Acquisition Diagnostic Tool," Transactions on Instrumentation and Measurement, vol. 45, no. 3, June 1996.

[20] J. Blair, "A Method for Characterizing Waveform Recorder Errors Using the Power Spectral Distribution," Transactions on Instrumentation and Measurement, vol. IM-41, no. 5, October 1992. 